\title{
Combined Core Needle Biopsy and Fine Needle Aspiration Cytology Using Returned Cell Block Method is Effective for Diagnosis of Cystic Lesions of the Breast
}

Taijiro Kosaka1, Shiho Azami², Mizuki lino², Yuuji Aoki², Kanako Ogura², Toshiaki Kitabatake ${ }^{3}$, Kuniaki Kojima ${ }^{3}$ and Toshiharu Matsumoto ${ }^{2 *}$ ${ }^{1}$ Department of Breast Surgery, Sainokuni Higashiomiya Medical Center, Japan

${ }^{2}$ Department of Diagnostic Pathology, Juntendo University Nerima Hospital, 3-1-10 Takanodai, Nerima-ku, Tokyo 177-8521, Japan

${ }^{3}$ Department of Breast Surgery, Juntendo University Nerima Hospital, Tokyo, Japan

\begin{abstract}
Background: Core needle biopsy (CNB) and fine needle aspiration cytology (FNAC) are useful methods to diagnose cystic lesions of the breast. The first English article describing the returned cell block method (RCBM: cell block from a Papanicolaou staining smear on a glass slide) was reported by us, and we also reported the usefulness of RCBM for the FNAC-based evaluation of tumors of the breast. In the present study, diagnostic procedures of CNB and FNAC using RCBM for cystic lesions of the breast were studied.
\end{abstract}

Methods: Forty-seven patients with cystic lesions of the breast diagnosed by sonographic examination and sonographically-guided FNAC were evaluated. In all cases, the evaluation of FNAC was performed by Papanicolaou staining smears, and RCBM was added in cases that were difficult to evaluate. The data from CNB (26 cases) and surgery ( 15 cases) were also evaluated.

Results: The accuracy of diagnosing carcinoma of FNAC from Papanicolaou staining smear was $56.2 \%$. The diagnostic accuracy increased to $75 \%$ on using RCBM, and the same level of accuracy was found on CNB. In 4 cases, a diagnosis of carcinoma was made by CNB only (2 cases) or FNAC using RCBM only (2 cases). Subsequently, the accuracy increased to $87.5 \%$ due to the combination of CNB and FNAC using RCBM.

Conclusions: Combined CNB and FNAC using RCBM is effective for the diagnosis of cystic lesions of the breast. Based on this new finding, we propose a procedure to diagnose cystic lesions of the breast by CNB and FNAC using RCBM.

Keywords: Core needle biopsy; Fine needle aspiration cytology; Returned cell block method; Cystic tumors of the breast; Diagnostic procedure

\section{Introduction}

Cystic lesions of the breast are common findings on mammography, ultrasonography, and magnetic resonance imaging. So, the evaluation of sonographic findings is important for diagnosis, treatment, and follow-up. The sonographic evaluation of such lesions has been reported based on the Breast Imaging Reporting and Data System (BIRADS) and various types of sonographic findings of cystic lesions of the breast have been proposed [1-5]. An excellent review of the literature on breast cystic masses was published by Berg, et al., and they described the management of various types of cystic lesion with the adoption of aspiration cytology and biopsy [4]. Until now, the published articles concerning cystic lesions of the breast have described the necessity of aspiration cytology and biopsy in cases with suspicious of malignancy [1-5]. However, a detailed study comparing aspiration cytology and biopsy for cystic lesions of the breast has not been reported, and so a decision on the practical way to use both methods is needed for the diagnosis of such lesions.

The authors initially published an English article describing the returned cell block method (RCBM: cell block from a Papanicolaou staining smear on a glass slide), and also reported the usefulness of RCBM for the fine needle aspiration cytology (FNAC)-based evaluation of tumors of the breast in 2016 [6].

The present study compares the diagnostic accuracy between core needle biopsy (CNB) and FNAC with or without RCBM in patients with cystic lesions of the breast. According to the results of this examination, a practical method using CNB and FNAC with RCBM for the diagnosis of cystic lesions of the breast is determined.

\section{Materials and Methods}

Among the patients undergoing ultrasonographically-guided FNAC examination of mammary lesions from January 1, 2012 to December 31, 2013 in Juntendo University Nerima Hospital, 138 with FNAC findings of a cyst, papilloma, or papillary carcinoma were selected. All patients were examined with both ultrasonography and mammography, and so a detailed re-evaluation of the sonographic findings from both examinations was done. Subsequently, 47 patients with definite cystic lesions were selected and examined. All of the examined patients were female and their ages ranged from 30 to 87 years (average, 57 years).

The sonographic findings in the 47 patients were evaluated according to BI-RADS [5]: simple cyst (Figure 1; 7 cases), complicated

*Corresponding author: Toshiharu Matsumoto, M.D., Department of Diagnostic Pathology, Juntendo University Nerima Hospital, 3-1-10 Takanodai, Nerima-ku, Tokyo 177-8521, Japan, Tel: +81-3-5923-3111; Fax: +81-3-5923-3217; E-mail: machian@juntendo.ac.jp

Received June 02, 2017; Accepted June 20, 2017; Published June 30, 2017

Citation: Kosaka T, Azami S, lino M, Aoki Y, Ogura K, et al. (2017) Combined Core Needle Biopsy and Fine Needle Aspiration Cytology Using Returned Cell Block Method is Effective for Diagnosis of Cystic Lesions of the Breast. J Cytol Histol 8: 462. doi: 10.4172/2157-7099.1000462

Copyright: ( 2017 Kosaka T, et al. This is an open-access article distributed under the terms of the Creative Commons Attribution License, which permits unrestricted use, distribution, and reproduction in any medium, provided the original author and source are credited. 


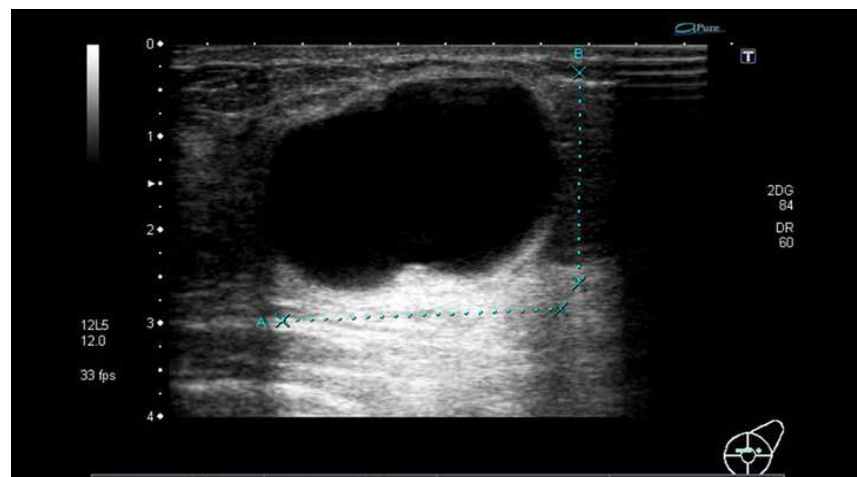

Figure 1: Sonogram of simple cyst. The shape of a cyst is oval with gently lobulated, the margin is circumscribed, the echo pattern is an echoic and there is acoustic enhancement.

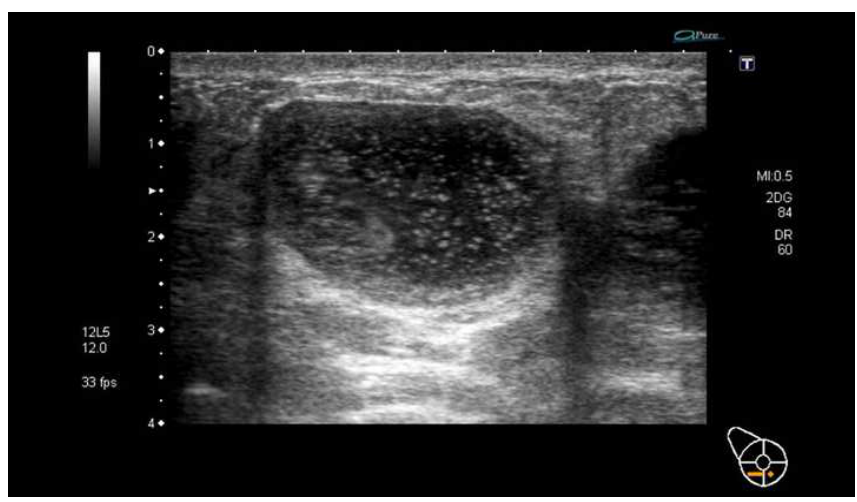

Figure 2: Sonogram of complicated cyst. A cyst shows homogeneous low-level internal echo throughout the lesion, containing fluid debris.

cyst (Figure 2; 24 cases), complex cyst (Figure 3; 15 cases), and unclassified cyst ( 1 case). These 4 types were compared with a benign or malignant group according to the cytological and histological diagnoses.

In all cases, the evaluation of FNAC by Papanicolaou staining smear was performed, and RCBM was added in cases that were difficult to evaluate with Papanicolaou staining smear. RCBM was described in our previous article, and it consists of making a cell block from a Papanicolaou staining smear on a glass slide, cutting sections of the block, and staining with conventional (HE stain and others) and immunocytochemistry stains [6]. According to the using of RCBM, easily recognition of morphology and application of immunocytochemistry can be made in the atypical cell clusters observed in a Papanicolaou staining smear [6]. In 6 patients undergoing repeated FNAC examinations, the findings of repeated FNAC were also evaluated.

For 26 patients, CNB examination was performed. For 15 patients, an operation, which consisted of tumorectomy (2 cases), partial resection of the breast (12 cases) and mastectomy (1 case), was performed. The data on the histological findings from CNB and surgical materials were reviewed. The pathological diagnosis by $\mathrm{CNB}$ and surgical materials was based on the newly published WHO classification of tumors of the breast [7]. The follow-up data on the examined cases were added to assess the benignity or malignancy of cases without histological examination.
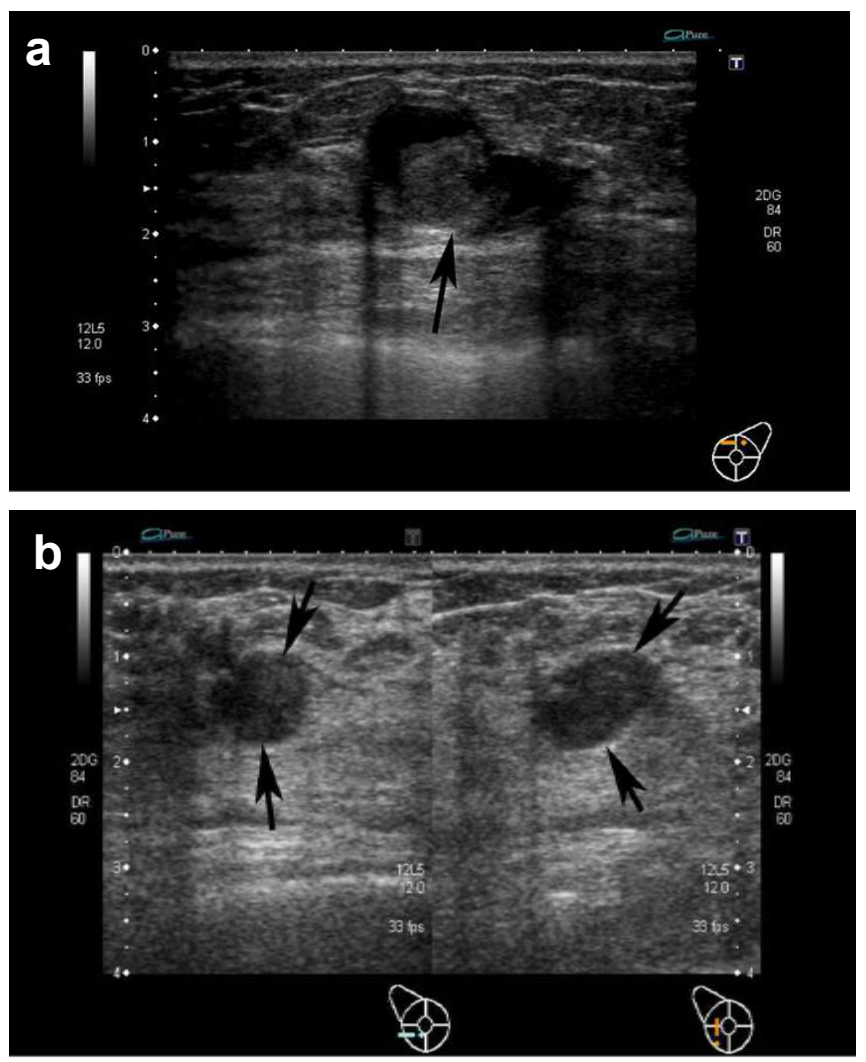

Figure 3: ( $a$ and b) Sonogram of complex cyst. Two patterns of the cyst, including intracystic mass (3a: indicated by an arrow) and intracystic discrete solid component (3b: indicated by arrows), are shown.

This study was approved by the Research Ethics Committee of Juntendo University Nerima Hospital. The surgeons obtained a written informed consent for the approval of cytological and histological examinations from all patients when they were examined with FNAC and CNB and they were operated. The approval of the opening of the data was not obtained from all of the patients, so personal information to pursue each of the patients was not included in the manuscript.

\section{Results}

Simple cysts according to BI-RADS were observed in the benign group only, and complicated and complex cysts were found in both groups of benignity and malignancy (Table 1). The diagnostic accuracy increased with the addition of RCBM compared with Papanicolaou staining smear only (Table 2 and Figure 4 ).

Comparisons between FNAC and CNB in 11 patients diagnosed with a carcinoma based on the surgical materials are shown in Table 3. In 5 patients, a diagnosis of carcinoma was made on both $\mathrm{CNB}$ and FNAC from Papanicolaou staining smear only (case no. 7-11; Figure 5). In 2 patients, carcinoma was diagnosed by FNAC using RCBM, but in these cases the diagnosis of carcinoma could not be made by CNB (case no. 4 and 5; Figure 6). In 1 patient, carcinoma was diagnosed by $\mathrm{CNB}$, but the diagnosis of carcinoma could not be made by FNAC, even with the use of RCBM (case no.2). A comparison between FNAC and CNB in 5 patients diagnosed with carcinoma from CNB without surgery is shown in Table 4. In 4 patients (case no. 13-16), the diagnosis of carcinoma was made based on FNAC from Papanicolaou staining smears, but in 1 patient (case no. 12; Figure 7), a diagnosis of malignancy could not be made by FNAC. 
Citation: Kosaka T, Azami S, lino M, Aoki Y, Ogura K, et al. (2017) Combined Core Needle Biopsy and Fine Needle Aspiration Cytology Using Returned Cell Block Method is Effective for Diagnosis of Cystic Lesions of the Breast. J Cytol Histol 8: 462. doi: 10.4172/2157-7099.1000462

Page 3 of 5

\begin{tabular}{|l|c|c|c|c|}
\hline Group & $\begin{array}{c}\text { Simple } \\
\text { cyst }\end{array}$ & $\begin{array}{c}\text { Complicated } \\
\text { cyst }\end{array}$ & $\begin{array}{c}\text { Complex } \\
\text { cyst }\end{array}$ & $\begin{array}{c}\text { Unclassified } \\
\text { cyst }\end{array}$ \\
\hline Benignity (31 cases) & 7 cases & 18 cases & 6 cases & 0 \\
\hline $\begin{array}{l}\text { Malignancy } \\
\text { (16 cases) }\end{array}$ & 0 & 6 cases & 9 cases & 1 case \\
\hline
\end{tabular}

BI-RADS: Breast Imaging Reporting and Data System.

Table 1: BI-RADS findings in benign and malignant groups of 47 patients with cystic lesions of the breast.

\begin{tabular}{|l|c|c|}
\hline FNAC diagnosis & Pap & Pap+RCBM \\
\hline Benignity & 28 cases $(59.5 \%)$ & 29 cases $(61.7 \%)$ \\
\hline Undetermined & 10 cases $(21.2 \%)$ & 6 cases $(12.7 \%)$ \\
\hline Malignancy & 9 cases $(19.1 \%)$ & 12 cases $(25.5 \%)$ \\
\hline
\end{tabular}

FNAC: Fine Needle Aspiration Cytology, Pap: Papanicolaou Staining Smear, RCBM: Return Cell Block Method. Incidence (expressed as a percentage) is the number of patients with each diagnosis per total number of examined patients.

Table 2: FNAC diagnosis based on Papanicolaou staining smear with or without RCBM in 47 patients with cystic lesions of the breast.
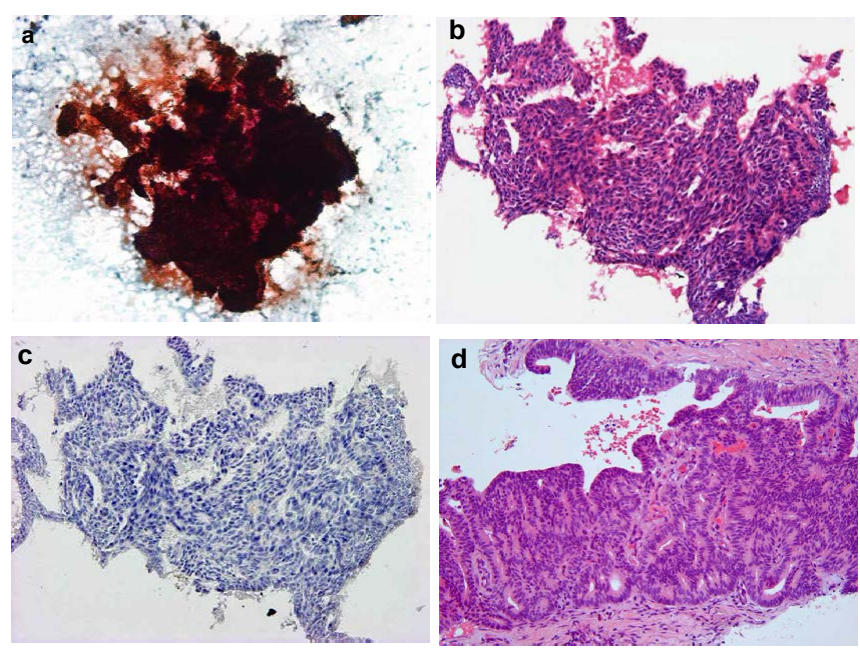

Figure 4: (a) Carcinoma is diagnosed by FNAC using RCBM and CNB (Case 6). (4a-4c: FNAC using RCBM) Note thick-layered cell clusters with papillary structure and nuclear enlargement of epithelial cells (Papanicolau stain, x100). (b) Papillary structure and cribriform pattern of epithelial cells with cellular atypia of moderate to severe (RCBM; HE stain, x200). (c) Disappearance of myoepithelial cells indicating papillary carcinoma (RCBM; p63 immunocytochemistry stain, x200). (4d) CNB: Note papillary and tubular structure of epithelial cells with cellular atypia of moderate to severe without myoepithelial cells in the cyst wall indicating intracystic papillary carcinoma (HE stain, $x 200)$.

The summary of the diagnosis of carcinoma from FNAC and CNB is shown in Table 5. The diagnostic accuracy of FNAC from Papanicolaou staining smear only was $56.2 \%$. The diagnostic accuracy increased to $75 \%$ on using RCBM, and the same accuracy was found with $\mathrm{CNB}$. Moreover, the accuracy increased to $87.5 \%$ on combining $\mathrm{CNB}$ and FNAC using RCBM.

\section{Discussion}

The correlation between sonographic subtypes and the pathological diagnosis has been studied [1-5,8]. Subsequently, the simple cyst subtype is almost always benign and routine follow-up screening is recommended [8]. On the other hand, non-simple cyst subtypes are not defined as benign and if there are findings suggesting malignancy, aspiration cytology and biopsy are recommended [8]. Chang et al. examined 175 sonographic cystic lesions using aspiration cytology

\begin{tabular}{|l|l|l|l|l|}
\hline $\begin{array}{l}\text { Case } \\
\text { No. }\end{array}$ & $\begin{array}{l}\text { FNAC } \\
\text { diagnosis by } \\
\text { Pap }\end{array}$ & $\begin{array}{l}\text { FNAC diagnosis by } \\
\text { Pap+RCBM }\end{array}$ & $\begin{array}{l}\text { Diagnosis by } \\
\text { CNB }\end{array}$ & $\begin{array}{l}\text { Diagnosis } \\
\text { surgical material }\end{array}$ \\
\hline 1 & Papillary lesion & Papillary lesion & Papillary lesion & ICPC \\
\hline 2 & Papillary tumor & Papillary tumor & ICPC & ICPC \\
\hline 3 & Papillary tumor & Papillary tumor & MC, suspected & ICPC \\
\hline 4 & Papillary tumor & PC & Fibrosis & IDC with ICPC \\
\hline 5 & Papillary tumor & PC & Papillary lesion & ICPC \\
\hline 6 & Papillary tumor & PC & ICPC & DCIS \\
\hline 7 & ICPC & RCBM, not performed & IDC & IDC \\
\hline 8 & PC & RCBM, not performed & ICPC & ICPC with DCIS \\
\hline 9 & IDC & RCBM, not performed & IDC & IDC and ICPC \\
\hline 10 & Carcinoma & RCBM, not performed & IDC & ICPC with IDC \\
\hline 11 & DCIS & RCBM, not performed & ICPC & IDC \\
\hline
\end{tabular}

*Assessment could not be conducted because of insufficient epithelial component in RCBM.

${ }^{* *}$ ICPC was removed by biopsy and DCIS around ICPC was present.

FNAC: Fine Needle Aspiration Cytology, Pap: Papanicolaou Staining Smear RCBM: Return Cell Block Method, CNB: Core Needle Biopsy, ICPC: Intracystic Papillary Carcinoma, MC: Mucinous Carcinoma, PC: Papillary Carcinoma, IDC Invasive Ductal Carcinoma, DCIS: Ductal Carcinoma In situ.

Table 3: Comparison among FNAC diagnosis based on Papanicolaou staining smear with or without RCBM and histological diagnosis of CNB in the 11 patients with mammary carcinoma with cystic lesions confirmed by the histological evaluation of surgical material.

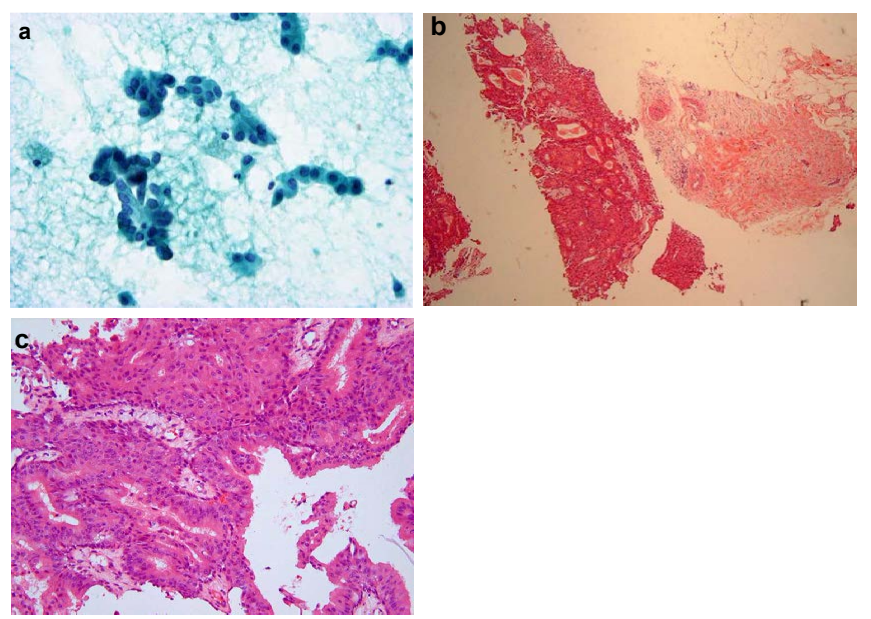

Figure 5: Carcinoma is diagnosed by FNAC and CNB (Case 8). (5a: FNAC) Papillary epithelial cell clusters with moderate cellular atypia indicating papillary carcinoma (Papanicolau statin, $x 400$ ). (5b and 5c: CNB) Note fibrous cyst wall and cellular epithelial tissue in $5 \mathrm{~b}$. Papillary and tubular epithelial components with moderate cellular atypia and without admixture of myoepithelial cells indicating intracystic papillary carcinoma in $5 c$ (HE stain; 5b, x40, 5c, x200).

and biopsy, and demonstrated malignancy in 40 patients showing a complicated solid and cystic mass or cyst with a thick wall/septa or nodule [2]. Berg et al. examined 150 sonographic cystic lesions using aspiration cytology only (55 cysts) and CNB only (81 cysts) and both aspiration and CNB (14 cysts), and demonstrated malignancy in 18 cysts showing a mass with a thick wall and/or thick septum, or intracystic mass, mixed and solid, or a solid mass with eccentric cystic foci [1]. In both studies by Chang et al. and Berg et al. [1,2], a comparison between aspiration cytology and biopsy regarding the accuracy of the diagnosis of malignancy was not performed.

Daly et al. examined 243 patients with sonographic complicated cysts and reported that one of them $(0.4 \%)$ was diagnosed with malignancy by aspiration cytology [3]. In 3 cases, atypical cells were 

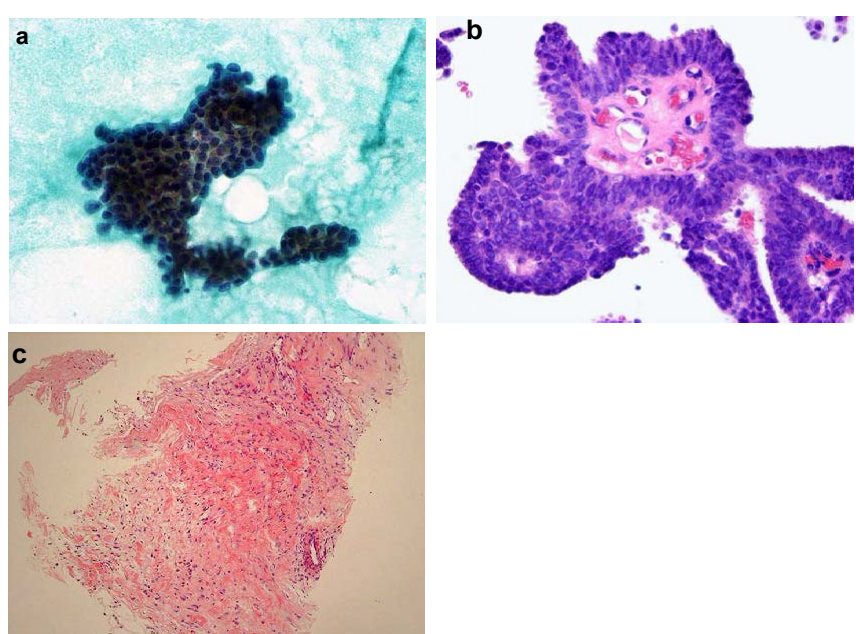

Figure 6: Carcinoma is diagnosed by FNAC using RCBM, but not by CNB (Case 4). (6a and 6b: FNAC using RCBM) Note thick layered papillary cell cluster, with mild to moderate cellar atypia, indicating papillary tumor in 6 a. (Papanicolau stain, x200). Papillary atypical epithelial cells with disappearance of myoepithelial cells, indicating papillary carcinoma in 6b (RCBM; HE stain, $\mathrm{x} 200)$. (6c: CNB) Note fibrosis without tumor cells (HE stain, $x 100$ ).

\begin{tabular}{|c|c|c|}
\hline Case No. & FNAC diagnosis by Pap* & Diagnosis by CNB \\
\hline 12 & Cystic disease & MC \\
\hline 13 & ICPC & ICPC \\
\hline 14 & PC & IDC \\
\hline 15 & IDC & ICPC \\
\hline 16 & IDC & IDC \\
\hline
\end{tabular}

*Return cell block method was not performed in all cases.

FNAC: Fine Needle Aspiration Cytology, CNB: Core Needle Biopsy, Pap: Papanicolaou Staining Smear, MC: Mucinous Carcinoma, ICPC: Intracystic Papillary Carcinoma, PC: Papillary Carcinoma, IDC: Invasive Ductal Carcinoma.

Table 4: Comparison among FNAC diagnosis based on Papanicolaou staining smear and histological diagnosis of CNB in the 5 patients with mammary carcinoma with cystic lesions without surgery.

\begin{tabular}{|l|c|}
\hline Methods & Diagnosis of carcinoma \\
\hline FNAC (Pap) & 9 cases $(9 / 16=56.2 \%)$ \\
\hline FNAC (Pap+RCBM) & 12 cases $(12 / 16=75 \%)$ \\
\hline CNB & 12 cases $(12 / 16=75 \%)$ \\
\hline FNAC (Pap+RCBM) and CNB & 14 cases $(14 / 16=87.5 \%)$ \\
\hline
\end{tabular}

FNAC: Fine Needle Aspiration Cytology, Pap: Papanicolaou Staining Smear, RCBM: Return Cell Block Method, CNB: Core Needle Biopsy.

Table 5: Diagnostic accuracy of FNAC with or without RCBM and CNB in the 16 patients with mammary carcinoma with cystic lesions.

observed in aspiration cytology, but in all cases the lesions were benign on surgical excision [3]. From this study, biopsy may be superior to aspiration cytology. Consistent with this, in the present study, the accuracy rate of $\mathrm{CNB}(75 \%)$ was greater than that of FNAC using Papanicolaou staining smear only (56.2\%). However, on using RCBM, the accuracy rate of FNAC increased to $75 \%$, and 2 patients were diagnosed with carcinoma using this method, but these patients could not be diagnosed with carcinoma by $\mathrm{CNB}$. On the other hand, in 2 patients, carcinoma was diagnosed by CNB only. Subsequently, the accuracy rate of diagnosing malignancy on combining CNB and FNAC using RCBM reached to $87.5 \%$. The results of the present study indicate that a combination of CNB and FNAC using RCBM is effective for the diagnosis of cystic lesions of the breast.

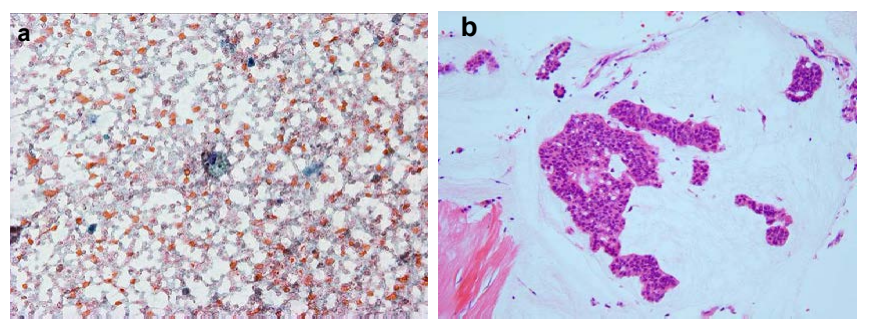

Figure 7: Carcinoma is diagnosed by CNB, but not by FNAC (Case 12). (7a: FNAC) Note presence of macrophage with hemorrhagic background indicating cystic disease (Papanicolau statin, x200). (7b: CNB) Floating of epithelial cell clusters with moderate cellular atypia within mucous lake indicating mucinous carcinoma, CNB (HE stain, x200)

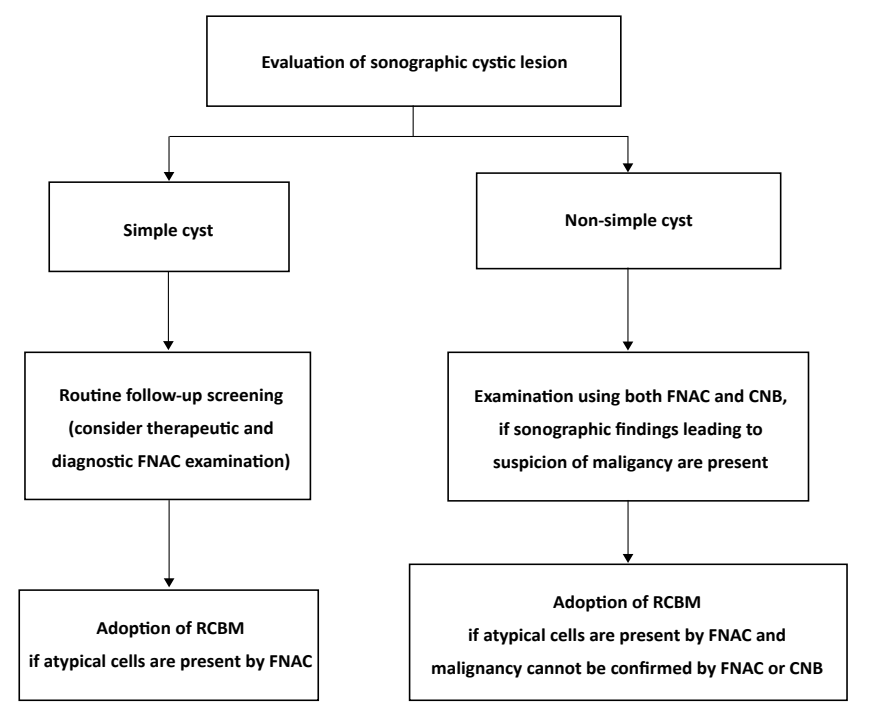

Figure 8: Algorithm of the procedures to diagnose cystic lesions of the breast by core needle biopsy (CNB) and fine needle aspiration cytology (FNAC) using the return cell block method (RCBM).

Intracystic papilloma and intracystic papillary carcinoma are common neoplasms showing cystic changes among mammary tumors [9]. In the present study, such tumors were examined in detail. On the other hand, as a rare neoplasm presenting with cystic changes in a breast tumor, squamous cell carcinoma is considered [10]. Squamous cell carcinoma of the breast usually presents as a cystic lesion and the cystic cavity is lined by squamous cells with various degrees of nuclear atypia and pleomorphism [10]. The neoplastic cells infiltrate the adjacent stroma and an increase in size occurs on follow-up. Among the reported cases of squamous cell carcinoma of the breast, the diagnosis using FNAS was different: benignity or malignancy $[11,12]$. This difference was caused by the difficultly in the evaluation of the atypia of squamous epithelium, and so if RCBM is used for such tumors, it provides a precise evaluation of the atypical status of squamous epithelium and relationship between the atypical epithelium and stroma, leading to a precise diagnosis.

\section{Conclusion}

Based on the results of the present study, we devised the algorithm for the diagnosis by CNB and FNAC using RCBM for cystic lesions of the breast (Figure 8). In the algorithm, the division of simple cyst and non-simple cysts is initially performed according to the findings 
of sonographic examinations. In a simple cyst, FNAC becomes a key point, so the presence of atypical cells in a Papanicolaou staining smear by FNAC is followed by the adoption of RCBM. On the other hand, in a non-simple cyst, both examinations of CNB and FNAC by Papanicolaou staining smear are conducted if sonographic findings indicate the possibility of malignancy. If atypical cells are present in Papanicolaou staining smear by FNAC and a diagnosis of malignancy cannot be made by FNAC and CNB, the adoption of RCBM is followed. Finally, we think that the proposed algorithm is optimal for diagnosis by CNB and FNAC for cystic lesions of the breast.

\section{Acknowledgements}

The authors thank Mr. D. Mrozek (Medical English Service, Kyoto, Japan) for organizing the English revision of this article.

\section{Conflicts of Interest}

The authors declare that there are no conflicts of interest.

\section{References}

1. Berg WA, Campassi $\mathrm{Cl}$, Loffe $\mathrm{OB}$ (2003) Cystic lesions of the breast: Sonographic-pathologic correlation. Radiology 227: 183-191.

2. ChangY-W, Kwon KH, Goo DE, Choi DL, Lee HK, et al. (2007) Sonographic differentiation of benign and malignant cystic lesions of the breast. J Ultrasound Med 26: 47-53

3. Daly CP, Bailey JE, Klein KA, Helvie MA (2008) Complicated breast cysts on sonography: Is aspiration necessary to exclude malignancy? Acta Radiol 15: 610-617.
4. Berg WA, Sechtin AG, Marques H, Zhang Z (2010) Cystic breast masses and the ACRIN 6666 experience. Radiol Clin North Am 48: 931-987.

5. Chen M, Zhan WW, Wang WP (2014) Cystic breast lesions by conventional ultrasonography: sonographic subtype-pathologic correlation. Arch Med Sci 10: $76-83$

6. Azami S, Aoki Y, Ogura K, Kojima K, Matsumoto T (2016) Application of returned cell block method (cell block from a Papanicolaou staining smear on a glass slide) for the evaluation of fine needle aspiration cytology of tumors of the breast. Diagn Cytopathol 44: 505-511.

7. Lakhani SR, Ellis IO, Schnitt SJ, Tan PH, van de Vijver MJ (2012) WHO classification of tumours of the breast. Lyon; IARC Press; 8-240.

8. Pearlman M, Griffin J, Swan M, Chelmow D (2016) Committee on Practice Bulletins-Gynecology in collaboration, Diagnosis and management of benign breast disorders. Obstet Gynecol 127: e141-e155.

9. O'Malley F, Visscher D, MacGrogan G, Tan PH, Ichihara S (2012) Intraductal papillary lesions. In: Lakhani SR, Ellis IO, Schnitt SJ, Tan PH, van de Vijver MJ (eds.) WHO classification of tumours of the breast. Lyon: IARC 99-109.

10. Reis-Fiho JS, Lakhani SR, Gobbi H, Sneige N (2012) Metaplastic carcinoma In: Lakhani SR, Ellis IO, Schnitt SJ, Tan PH, van de Vijver MJ (eds.) WHO classification of tumours of the breast. Lyon: IARC 48-52.

11. Vera-Alvarez J, Garcia-Prats MD, Marigil-Gómez M, Abascal-Agorreta $M$ López-López JI, et al (2007) Primary pure squamous cell carcinoma of the breast diagnosed by fine-needle aspiration cytology: a case study using liquidbased cytology. Diagn Cytopathol 35: 429-432.

12. Ramos V, Fraga J, Simöes T, Figueiredo Dias M (2016) Intracystic primary squamous cell carcinoma of the breast: A challenging diagnosis. Case Rep Obstet Gynecol 2016:6081634. Epub 2016 Sep 26. 